\title{
Global condensation: a "climate change" towards better standardisation of exhaled breath condensate measurements
}

\section{To the Editors:}

We read with great interest the letter by LiU et al. [1] and fully agree that exhaled breath condensate (EBC) collection devices significantly affect EBC biomarker levels, and that absolute values from different devices are not directly comparable [1, 2]. We also agree that one single (currently available) collection device may not be ideal for all applications and that, in future studies, each marker may need to be tested by a variety of devices in order to determine the optimal collection apparatus [2]. In our opinion, some additional considerations of EBC methodology should be made. We believe that these may change our current view on the variability associated with the measurement of exhaled markers in EBC. In turn, this altered consciousness may provide a tentative way out of current methodological controversies and, by consequence, it may offer new perspectives towards the standardisation of EBC methodology [3].

First, the levels of nitrogen oxides and total protein measured by LIU et al. [1] in EBC were expressed as nmol or $\mu \mathrm{g}$ "per $10 \mathrm{~min}^{\prime}$, and may suggest an influence of the duration of EBC collection on the concentration of these biomarkers in EBC. In fact, we also believe that such an influence exists; however, the question is whether standardisation for time results in the best reproducibility. So far, no study has answered this, whereas some studies have standardised for EBC volume or a dilution factor [4]. Alternatively, measurements can be standardised for the volume of exhaled air, which resulted in significant correlations between exhaled nitrite and leukotriene $\mathrm{B}_{4}$ in the study of KNOBLOCH et al. [5] in calves. In the data reported by LIU et al. [1], EBC was collected during a fixed period of $10 \mathrm{~min}$ in each tidally breathing subject, by each of the different collection systems. Therefore, it seems logical to report the level of biomarkers in the resulting condensate in relation to this period of time. However, this bypasses an important methodological EBC collection issue, as it does not take into account that each of these devices are open-ended systems. In an open-ended collection system, the available condensation surface will be saturated after a certain period of time and thus, before and beyond this point in time, noncondensed exhaled breath may be lost through the open end of the collection device. By consequence, exhaled respiratory droplets with their possible solutes (or biomarkers) can also be lost. For this reason, there is in fact an influence of the duration of collection on the concentration of biomarkers in EBC, although we should consider this as an influence of the condensation saturation point of each device for each single subject. As confirmation of this theoretical consideration, we found in several studies that the volume of collected EBC within a certain period of time is highly variable, which makes standardisation for time not optimal, in our opinion.
Therefore, we believe, this issue may only be solved through use of a closed collection system that enables "global condensation", i.e. the condensation of the total exhaled breath volume within a certain period of time [6]. As it is now evident from several studies that collection devices can influence EBC marker concentration, the time has come to unravel which factors are mainly responsible for this effect: dimensions of the device, condensation surface, temperature of the condenser tube, closed or open design, and so on [1, 2, 7-9].

Secondly, we agree with LIU et al. [1] that it is essential for EBC methodology to have reproducibility data on (all) biomarkers in (all) collection devices. Moreover, we even suggest that it is essential to have reproducibility data on each biomarker, both per specific assay, as well as per collection device. Furthermore, we should be careful when interpreting data from only six healthy subjects. Likewise, total protein measurements, a rough value for the total protein content in a biofluid, were performed using an assay with a lower limit of detection of $4 \mu \mathrm{g} \cdot \mathrm{mL}^{-1}$, and thus one should be equally careful when interpreting median values of $6.6 \pm 3.3 \mu \mathrm{g}$ total protein [1]. Furthermore, and with respect to the variability of markers in $\mathrm{EBC}$, it may be that the reproducibility of markers can be improved by standardising for exhaled volume, time or a dilution factor [10]. In fact, we urgently need more studies to address this important topic.

Thirdly, regarding $\mathrm{pH}$ in $\mathrm{EBC}$, we would like to refer to the study of PRIETO et al. [9] who demonstrated higher values of deaerated and nondeaerated $\mathrm{pH}$ using the EcoScreen compared with the Rtube.

Taken together, it is evident from several studies that exhaled breath condensate collection devices have an influence on concentrations of biomarkers in exhaled breath condensate [1, 2, 7-9]. However, the key questions remain: which device characteristics are mainly responsible for this effect? And what are the optimal device characteristics? Moreover, it is important to study the influence of different designs of exhaled breath condensate collection devices on biomarker reproducibility for each group of markers (cytokines, nitrogen oxides, markers of oxidative stress, leukotrienes and so on) in sufficiently large groups of subjects, with or without disease. We believe that this "climate change" may offer new perspectives towards a better standardisation of exhaled breath condensate measurements.

P.P.R. Rosias*\#, Q. Jöbsis*, K. van de Kant*, C. Robroeks*, C.P. van Schayck ${ }^{\star}$, L.J.I. Zimmermann ${ }^{+}$and E. Dompeling* *Dept of Paediatric Pulmonology, Care and Public Health Research Institute, University Hospital Maastricht, "Dept of General Practice, Care and Public Health Research Institute, Maastricht University, ${ }^{+}$Dept of Paediatrics, University 
Hospital Maastricht, Maastricht, and "Dept of Paediatrics, Maasland Hospital, Sittard, the Netherlands.

\section{STATEMENT OF INTEREST}

None declared.

\section{REFERENCES}

1 Liu J, Conrad DH, Chow S, Tran VH, Yates DH, Thomas PS. Collection devices influence the constituents of exhaled breath condensate. Eur Respir J 2007; 30: 807-808.

2 Rosias PP, Robroeks CM, Niemarkt HJ, et al. Breath condenser coatings affect the measurement of biomarkers in exhaled breath condensate. Eur Respir J 2006; 28: 1036-1041.

3 Horváth I, Hunt J, Barnes PJ. Exhaled breath condensate: methodological recommendations and unresolved questions. Eur Respir J 2005; 26: 523-548.

4 Baker EH, Clark N, Brennan AL, et al. Hyperglycemia and cystic fibrosis alter respiratory fluid glucose concentrations estimated by breath condensate analysis. J Appl Physiol 2007; 102: 1969-1975.
5 Knobloch H, Becher G, Decker M, Reinhold P. Within the exhaled breath condensate, nitrite and $\mathrm{LTB}_{4}$ are correlated to each other when calculated per 100 litres exhaled breath. Eur Respir J 2006; 28: Suppl. 50, 789s.

6 Rosias P, van de Kant K, Robroeks C, Heynens J, Jöbsis R, Dompeling E. Feasibility of exhaled breath condensate collection in toddlers with and without recurrent wheeze. Eur Respir J 2007; 30: Suppl. 51, 365s.

7 Tufvesson E, Bjermer L. Methodological improvements for measuring eicosanoids and cytokines in exhaled breath condensate. Respir Med 2006; 100: 34-38.

8 Soyer OU, Dizdar EA, Keskin O, Lilly C, Kalayci O. Comparison of two methods for exhaled breath condensate collection. Allergy 2006; 61: 1016-1018.

9 Prieto L, Ferrer A, Palop J, Domenech J, Lusar R, Rojas R. Differences in exhaled breath condensate $\mathrm{pH}$ measurements between samples obtained with two commercial devices. Respir Med 2007; 101: 1715-1720.

10 Effros RM, Biller J, Foss B, et al. A simple method for estimating respiratory solute dilution in exhaled breath condensates. Am J Respir Crit Care Med 2003; 168: 1500-1505.

\section{Excessive daytime sleepiness in patients with sleep- disordered breathing}

\section{To the Editors:}

I read with interest the article by MEDIANO et al. [1] about excessive daytime sleepiness (EDS) in patients with obstructive sleep apnoea (OSA). In order to investigate the determinants of EDS in OSA patients, the authors compared the demographics and polysomnographic variables of two groups of OSA patients who had a similar apnoea/hypopnoea index (AHI), age and body mass index (BMI). Nocturnal hypoxaemia was reported to be a major determinant of EDS in patients with OSA.

I would like to point out that the possible coexistence of obesity hypoventilation syndrome (OHS), which is a known cause of EDS, was not ruled out in the studied group [2]. HIDA et al. [3] have shown that OHS patients have a significantly higher Epworth Sleepiness Scale score compared with pure OSA patients matched for age, BMI and AHI. It is well documented that $70-90 \%$ of patients with OHS have predominantly obstructive events during sleep [4]. Therefore, to rule out coexisting OHS, measurement of daytime blood gases and nocturnal monitoring of carbon dioxide arterial tension, as well as the observation for oxygen desaturation during sleep that is not explained by apnoeic or hypopnoeic events, become imperative.

MEDIANO et al. [1] reported that patients with EDS had lower nocturnal oxygen saturation and mean arterial saturation. However, the desaturation index was not reported. As patients with hypoventilation may have frequent episodes of desaturation without obstructive apnoeic or hypopnoeic events, it becomes important to compare the desaturation index in both groups. The group with EDS was found to have a longer apnoea duration, which the authors attributed to intrinsic differences in arousal thresholds or increased sleep pressure [1].

It is known that patients with OHS exhibit periods of sustained sleep hypoxaemia [5]. Sustained hypoxaemia has been suggested as a cause of delayed arousal from sleep [6], which may explain the longer apnoea duration in the EDS group. The fact that patients with and without EDS had a similar age, BMI and AHI does not imply that they have the same underlying sleepdisordered breathing [1]. A recent large study [7] reported no difference in AHI between OHS patients and pure OSA patients matched for BMI. In another recently published study [8], patients with $\mathrm{OHS}$ and hypercapnic respiratory failure were compared with patients with pure OSA matched to each subject using BMI, age and AHI measured at the time of diagnosis. Compared with pure OSA patients, OHS patients spent more time with arterial oxygen tension $<90 \%$, had lower mean saturation, a higher desaturation index and their lowest recorded oxygen saturation was significantly lower [8]. We agree with MEDIANO et al. [1] that nocturnal hypoxaemia may play a role in the pathogenesis of EDS; however, we feel that OHS was not ruled out as a cause of EDS in the studied group.

Data suggest that obesity hypoventilation syndrome is both under recognised and underdiagnosed [2]. To overcome these problems, the serum bicarbonate level should be measured in patients with sleep-disordered breathing, excessive daytime 